

\title{
EVALUACIÓN TÉRMICA DEL MATERIAL ISOBLOCK EN EL CLIMA CÁLIDO SECO DE CIUDAD JUÁREZ, MÉXICO
}

\author{
A THERMAL EVALUATION OF THE ISOBLOCK MATERIAL IN THE \\ WARM, DRY CLIMATE OF CIUDAD JUÁREZ, MEXICO
}

\author{
LUIS CARLOS HERRERA SOSA \\ Universidad Autónoma de Ciudad Juárez, \\ Instituto de Arquitectura, Diseño y Arte \\ Juárez, México \\ carlos.herrera@uacj.mx
}

\begin{abstract}
RESUMEN
Para que las viviendas mejoren su comportamiento térmico y disminuyan el consumo energético, producto del uso excesivo de sistemas de climatización convencional, las Normas de Eficiencia Energética de México establecen un factor de Resistencia Térmica ("R") mínima de acuerdo con cada región climática del país. Este documento presenta las conclusiones del trabajo de investigación térmica del material denominado Isoblock, que consiste en un block de concreto con aislamiento en su interior, que cumple satisfactoriamente con el factor " $R$ " solicitado por la normativa. La investigación busca demostrar que es necesario que la normativa citada contemple la masa térmica de los materiales dentro de los parámetros térmicos a considerar. Desde ese enfoque, la metodología consistió en comparar el lsoblock con un block de concreto convencional en dos módulos de iguales características durante las temporadas climáticas extremas en Ciudad Juárez, Chihuahua. Por último, se simuló térmicamente con el software Design Builder V4.5 y se correlacionaron los resultados para su validación. A partir de dichos resultados se simuló el módulo de Isoblock con mayor cantidad de masa térmica sin variar su espesor, para medir su impacto en la temperatura interior. Los datos obtenidos demuestran que un material con un mismo factor " $R$ " puede alcanzar temperaturas del aire interior más confortables, en temporadas extremas
\end{abstract}

\section{Palabras clave}

masa térmica, Isoblock, resistencia térmica

\begin{abstract}
In order for dwellings to improve their thermal behavior and reduce energy consumption due to the excessive use of conventional air heating and cooling systems, the Mexican Energy Efficiency Standards establish a minimum thermal resistance factor ("R") according to each climatic region of the country. This article presents the conclusions of thermal research on the material known as Isoblock,

which: consists of a concrete block with insulation inside and satisfactorily meets the " $R$ " factor required by the regulations. The study seeks to demonstrate the necessity of taking the thermal mass of materials into consideration within the thermal parameters in the aforementioned regulations. From that approach, the methodology consisted in comparing the Isoblock with a conventional concrete block in two modules with the same characteristics during the extreme seasons in Ciudad Juárez, Chihuahua. Lastly, a simulation was carried out using Design Builder V4.5 software and the results were correlated for validation. With these results, the Isoblock module was simulated with a greater amount of thermal mass without varying its thickness, to measure its impact on the interior temperature. The results obtained demonstrate that a material with the same " $R$ " factor can produce more comfortable indoor air temperatures in extreme seasons
\end{abstract}




\begin{tabular}{|c|c|c|c|c|c|c|c|c|c|}
\hline & \multicolumn{3}{|l|}{ Techos } & \multicolumn{3}{|l|}{ Muros } & \multicolumn{3}{|c|}{ Entrepisos ventilados } \\
\hline & Mínima & Habitabilidad & $\begin{array}{c}\text { Ahorro } \\
\text { de } \\
\text { energía }\end{array}$ & Mínima & Habitabilidad & $\begin{array}{c}\text { Ahorro } \\
\text { de } \\
\text { energía }\end{array}$ & Mínima & Habitabilidad & $\begin{array}{c}\text { Ahorro } \\
\text { de } \\
\text { energía }\end{array}$ \\
\hline 1 & 1.40 & 2.10 & 2.65 & 1.00 & 1.10 & 1.40 & NA & NA & NA \\
\hline $3 \mathrm{~A}$ & 1.40 & 2.30 & 2.80 & 1.00 & 1.23 & 1.80 & 0.90 & 1.40 & 1.60 \\
\hline $4 \mathrm{~A}$ & 1.40 & 2.65 & 3.20 & 1.00 & 1.80 & 2.10 & 1.10 & 1.80 & 1.90 \\
\hline
\end{tabular}

Tabla 1. Factor "R" requerido por zona climática. Elaboración del autor en base a la NMX-C-460-ONNCCE-2009. *unidades en $\mathrm{m} 2 \mathrm{~K} / \mathrm{W}$

\section{INTRODUCCIÓN}

A través de diferentes instituciones, México ha establecido normas de ahorro energético para las edificaciones que buscan la mitigación de los efectos del cambio climático y que promueven la sostenibilidad. Estas normas son producto de los compromisos adquiridos desde 1992 ante la Convención Marco de las Naciones Unidas sobre el Cambio Climático y fueron ratificadas ante la Organización de las Naciones Unidas en 1993. Hay considerar además, en este contexto, los acuerdos generados por el protocolo de Kioto, que entró en vigor el 16 de febrero de 2005 para las naciones que lo ratificaron, entre ellas México, que lo hizo en el año 2000 (SEMARNAT, 2016).

Se estima que en México el sector de vivienda emitió 75 millones de toneladas de $\mathrm{CO}_{2}$ sólo en el año 2006, lo que correspondió el $12 \%$ de las emisiones de este gas de efecto invernadero en el país (Comisión Nacional de Vivienda, 2010: 17). Asimismo, este sector representó el 16.2\% del consumo total de energía en el país (Fundación IDEA A.C., 2013: 28). Ahora bien, en el caso de la vivienda, hasta el año 2000 no se contaba con ninguna directriz de sostenibilidad o de eficiencia energética.

Desde el año 2001, México, a través de la Comisión Nacional para el Uso Eficiente de la Energía (CONNUE) de la Secretaría de Energía, ha emitido Normas Oficiales Mexicanas (NOM) en materia de eficiencia energética (NOM-ENER). Estas son formuladas por el comité Consultivo Nacional de Normalización para la Preservación y Uso racional de los Recursos Energéticos (Vallejo, 2014: 46). En este marco, que establece especificaciones técnicas de aplicación obligatoria, se encuentran la NOM020-ENER-2011 Eficiencia energética en edificaciones, envolvente de edificios para uso habitacional, y la NORMA MEXICANA NMX-C-460-ONNCCE-2009.

Tales normativas tienen como objetivo limitar las ganancias de calor de los edificios residenciales a través de su envolvente con el fin de racionalizar el uso de energía en los sistemas de enfriamiento (Normas Oficiales Mexicanas, 2015).

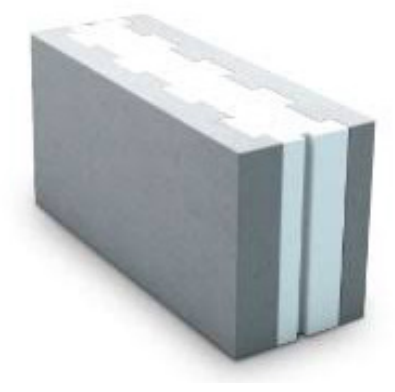

Figura 1. ISOBLOCK. Fuente: Grupo Cementos de Chihuahua, 2016.

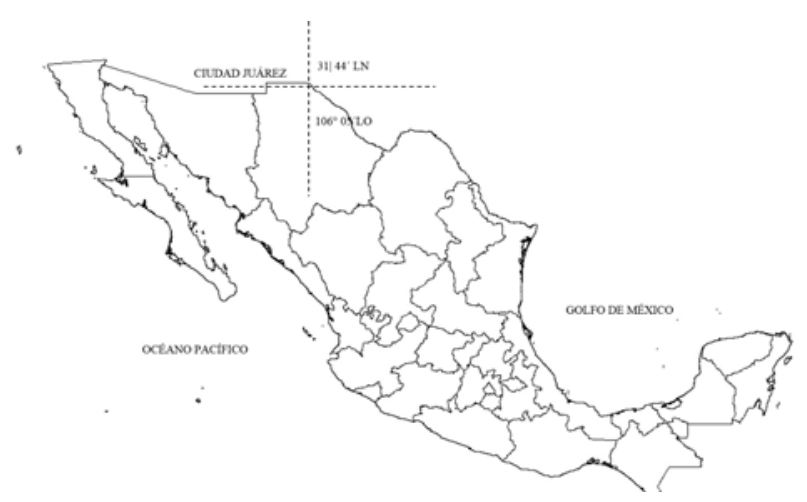

Figura 2. Localización Ciudad Juárez. Fuente. Elaboración del autor en base mapa de INEGI.

Ambas normas establecen un factor de Resistencia Térmica ("R") mínima de acuerdo a la zona térmica del país, para cada uno de los componentes de la envolvente en contacto con el exterior. El estado de Chihuahua contiene las zonas térmicas 1, 3A y 4A y, específicamente, Ciudad Juárez se localiza en la zona térmica 1 (ver Tabla 1).

Por ese motivo los constructores y desarrolladores de vivienda en Chihuahua han estado realizando diversas búsquedas de materiales que permitan cumplir con lo requerido. En ese sentido, el Grupo Cementos de 
Chihuahua, empresa líder en Chihuahua en la producción de cemento y concreto, ha elaborado un material denominado ISOBLOCK, el cual consiste en un bloque de concreto de 12 o $15 \mathrm{~cm}$ de espesor, $19.4 \mathrm{~cm}$ de alto y $39.6 \mathrm{~cm}$ de largo, que contiene en su centro $4.5 \circ 6.8 \mathrm{~cm}$ de aislamiento con poliestireno expandido de alta densidad, y a ambos lados concreto de 3.75 o $4.10 \mathrm{~cm}$, respectivamente (ver Figura 1).

La resistencia térmica del Isoblock de $12 \mathrm{~cm}$ de espesor es de $1.4147 \mathrm{~m}^{2} \mathrm{~K} / \mathrm{W}$ y la del de $15 \mathrm{~cm}$ de espesor es de $1.9005 \mathrm{~m}^{2} \mathrm{~K} / \mathrm{W}$. Ambos valores se encuentran por encima del mínimo requerido en muros de las zonas térmicas del estado de Chihuahua.

No obstante que cumplen con lo solicitado en las normas, estas no contemplan el efecto que tiene la inercia térmica (masa térmica) del concreto al interior de la edificación, el cual, por su calor específico volumétrico, es capaz de ampliar, de acuerdo al espesor del concreto, el desfase o tiempo de retraso y el amortiguamiento del material.

\section{OBJETIVOS}

El objetivo de este estudio es evaluar el impacto que tiene el uso del Isoblock en muros, en la temperatura del aire interior de un espacio y, más concretamente, evaluar el impacto de diferentes espesores de concreto en contacto con el interior del Isoblock en la temperatura interior de un espacio.

\section{METODOLOGÍA}

El proyecto de investigación se realizó en las instalaciones del Grupo Cementos de Chihuahua (GCC), ubicadas en Ciudad Juárez, Chihuahua; ubicación escogida por sus condiciones climáticas extremas en las temporadas de invierno y verano.

Ciudad Juárez se localiza al norte del Estado de Chihuahua en México, frontera con la Ciudad de El Paso, Texas en Estados Unidos. Delimita al norte con el Río Bravo, al poniente, con una sierra montañosa y al sur, con una extensión desértica de arenales. Sus coordenadas geográficas son $31^{\circ} 44^{\prime}$ latitud norte, $106^{\circ} 05^{\prime}$ longitud oeste y $1835 \mathrm{msnm}$ (ver Figura 2).

La ciudad se caracteriza por ser una zona semidesértica con un clima cálido seco extremoso, de veranos muy calurosos, que presentan temperaturas máximas extremas de hasta $46^{\circ} \mathrm{C}$, e inviernos muy fríos, con temperaturas mínimas extremas de $-27^{\circ} \mathrm{C}$ (Comisión Nacional del Agua, 2016). El promedio anual de humedad relativa es de $46 \%$, con humedades mínimas de $29 \%$ y máximas de hasta $80 \%$, en enero.

Con propósitos comparativos, se determinó la zona de confort para Ciudad Juárez de acuerdo a ANSI/ASHRAE Standard 552010, para verano e invierno, con el promedio de temperaturas medidas en campo durante el periodo de registro de datos:

$\mathrm{Tn}=17.8+0.31 *(\mathrm{Tamb})$

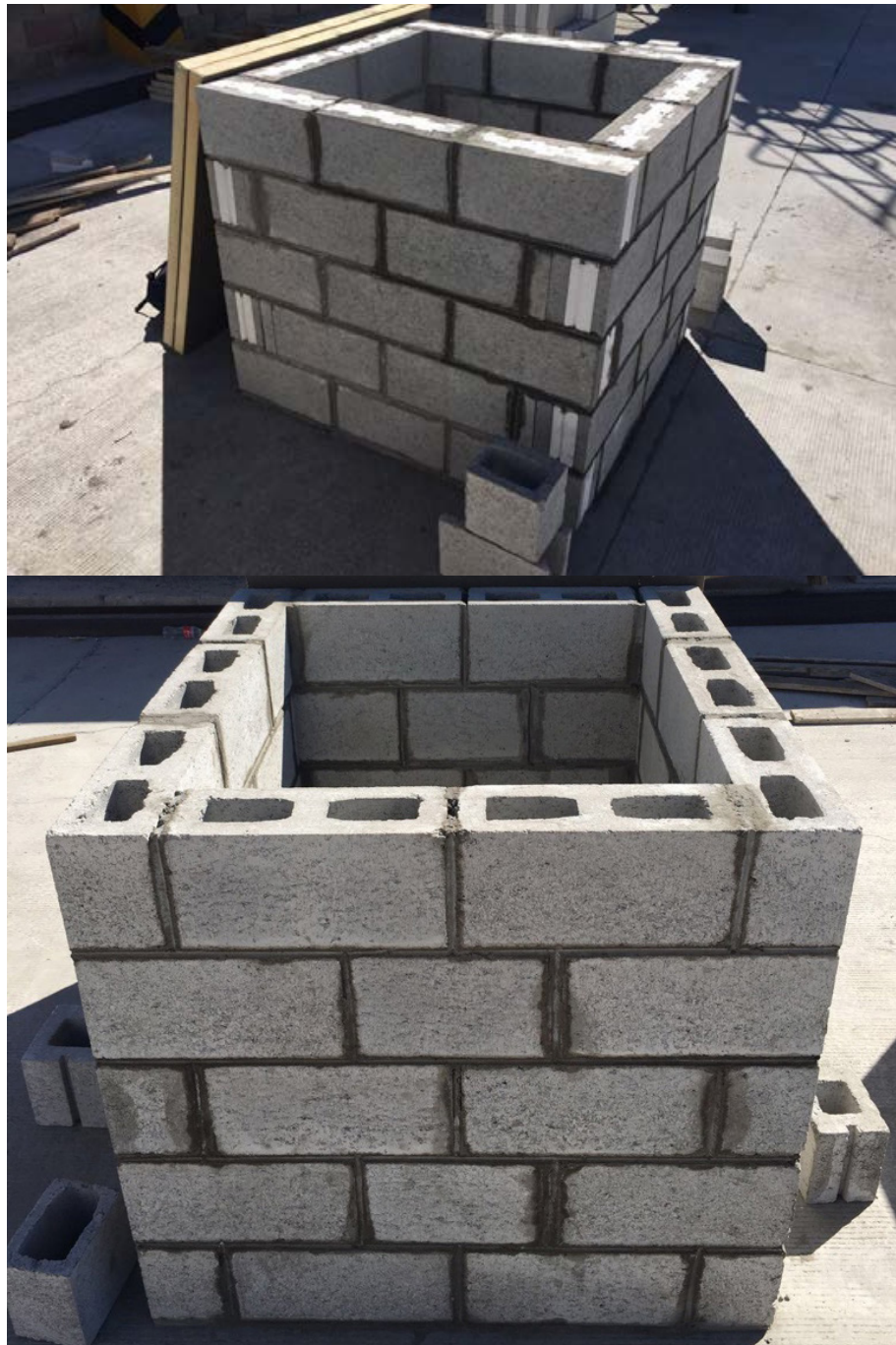

Figura 3a y 3b. Módulos de Isoblock y Block de concreto convencional. Fuente. Fotografías tomadas por el autor.

Donde:

$\mathrm{Tn}=$ Temperatura neutral

Tamb. $=$ Temperatura ambiente promedio

El resultado para la temporada de invierno fue de una temperatura neutral de $24.03^{\circ} \mathrm{C}$, con un límite superior de $26.53^{\circ} \mathrm{C}$ y uno inferior de $21.53^{\circ} \mathrm{C}$. Para la temporada de verano la temperatura neutral fue de $26.94^{\circ} \mathrm{C}$, con un límite superior de $29.44^{\circ} \mathrm{C}$ y un límite inferior de $24.44^{\circ} \mathrm{C}$.

\section{El proyecto de investigación se desarrolló en tres etapas:}

En la primera etapa se monitorearon en campo dos módulos durante una semana, tanto en la temporada de invierno o fría (febrero) como en la temporada de verano o caliente (junio). Ambos módulos se construyeron de $1 \mathrm{~m} \times 1 \mathrm{~m} \times 1 \mathrm{~m}$ con espesores de muro de $15 \mathrm{cms}$ orientados a cada uno de los puntos cardinales. Uno de los módulos se edificó con muros a base de Isoblock y el otro con block de concreto hueco convencional (ver Figuras 3a y 3b). 


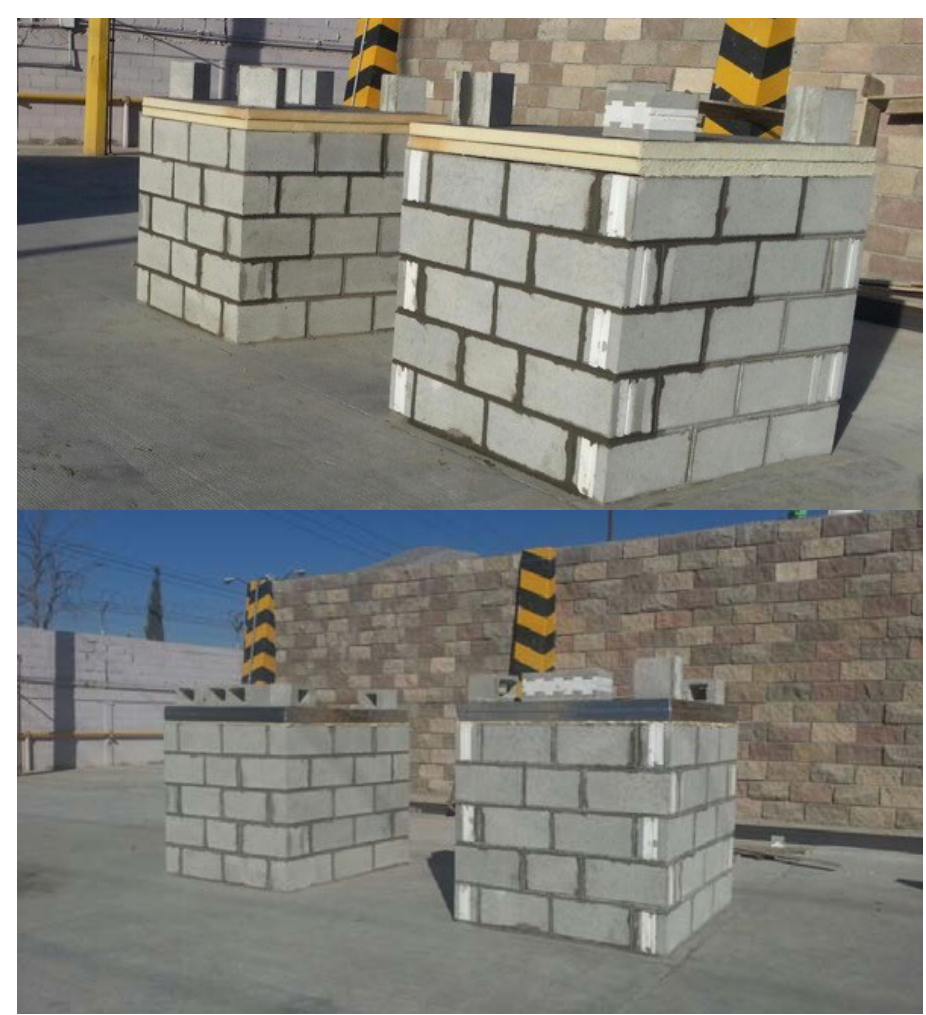

Figura 4a y 4b. Módulos de investigación. Fuente: Fotografías tomadas por el autor.

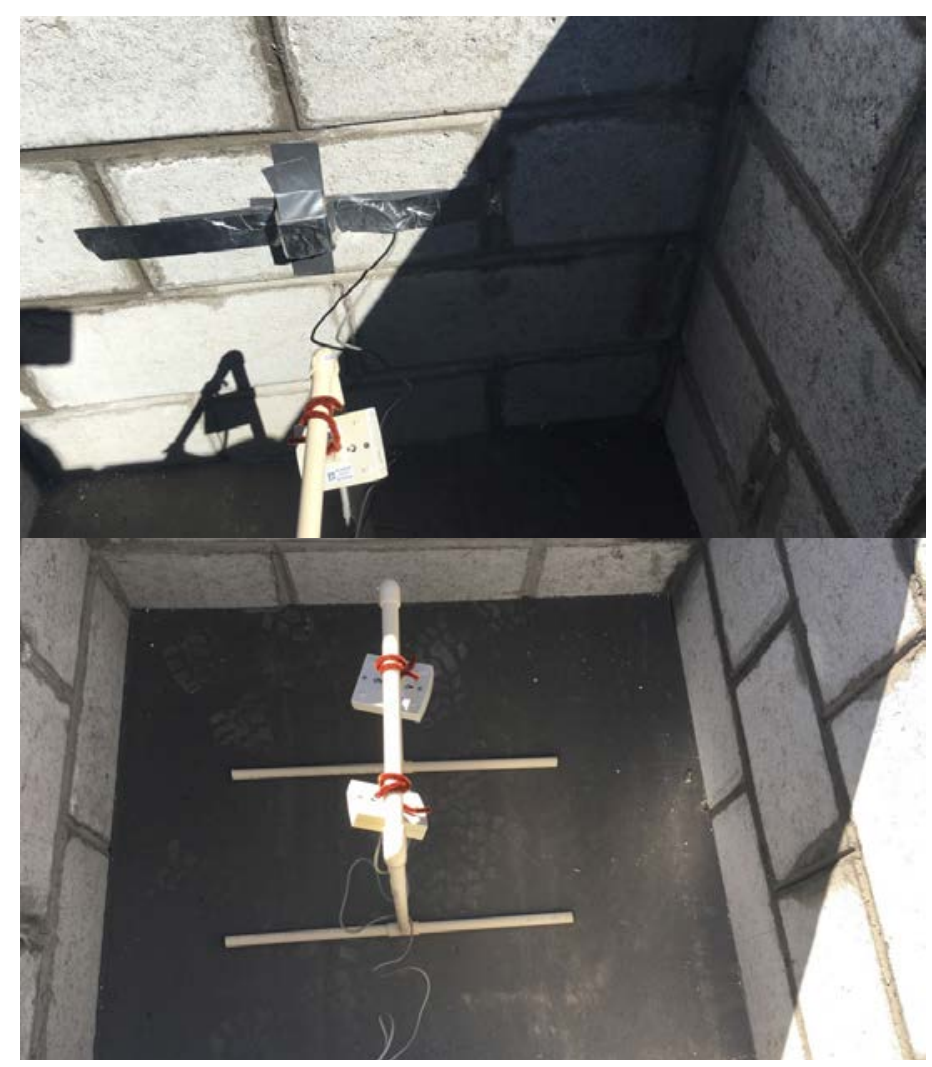

Figura 5a y 5b. Ubicación de data loggers tipo hobo. Fuente. Fotografías tomadas por el autor.
En la cubierta y en la parte en contacto con el piso se colocaron dos placas de aislante de polisocianurato de una pulgada de espesor cada una (ver Figuras 4a y $4 b$ ).

Las ganancias solares de los módulos se consideran a través de la temperatura del aire interior, pues al estar constituidos sin vanos el aporte se considera como ganancia solar por conducción con temperatura sol aire. Las ganancias internas no se consideran debido a que los módulos no están habitados para evitar variables durante el proceso de investigación.

En los módulos se colocaron sensores tipo Hobo U12 marca Onset para el registro de la temperatura del aire interior, humedad relativa y la temperatura superficial (temperatura de cable) del muro norte, cada 15 minutos. También se instaló un sensor de temperatura y humedad relativa tipo Hobo 008 marca Onset en el exterior. Estos data loggers cumplen con los requisitos de la ISO 7726:1998, Clase I (ver Figuras $5 a$ y $5 b$ ).

En la segunda etapa se realizaron simulaciones térmicas con el software Design Builder V4.5.

Al igual que en la etapa de campo, las simulaciones térmicas se realizaron con dos módulos de iguales características, con el registro de temperatura interior del aire y la temperatura exterior.

En la tercera etapa se efectuaron correlaciones de los resultados de las dos primeras etapas para validar resultados. Esto permitió, por un lado, conocer los efectos finales $y$, por otro, llevar a cabo simulaciones para medir el efecto de la masa térmica del material Isoblock con diferentes espesores en contacto con el interior.

\section{CASO DE ESTUDIO}

Pese a que la idoneidad técnica del Isoblock determina un valor de resistencia térmica de $1.9005 \mathrm{~m}^{2} \mathrm{~K} / \mathrm{W}$, se acordó, para objetivos de esta investigación, realizar el cálculo del factor "R" como se establece en la NMX-C-460ONNCCE-2009 (ver Figura 6).

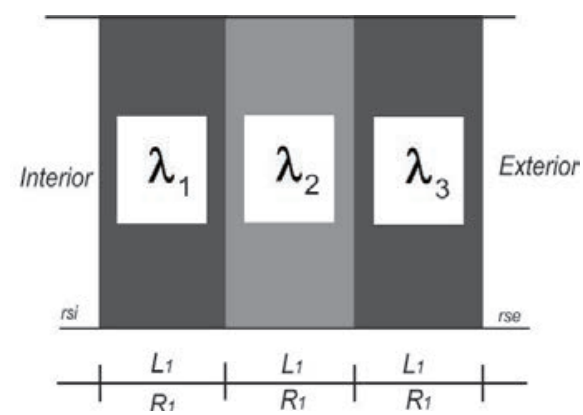

Figura 6. Cálculo de resistencia térmica. Fuente: Elaboración del autor en base a la NMX-C-460 ONNCE-2009. 


\begin{tabular}{|c|c|c|c|c|c|c|c|}
\hline & \multicolumn{9}{|c|}{ ISOBLOCK } \\
\cline { 2 - 8 } & EXTERIOR & INTERMEDIO & INTERIOR & \multicolumn{2}{|c|}{$\begin{array}{r}\text { FACTOR "R" } \\
\text { m2 K/W }\end{array}$} & \multicolumn{2}{|c|}{$\begin{array}{r}\text { FACTOR "U" } \\
\text { W/m2 }\end{array}$} \\
\cline { 2 - 8 } & CONCRETO & AISLANTE & CONCRETO & $\begin{array}{r}\text { DESIGN } \\
\text { BUILDER }\end{array}$ & $460-$-ONNCCE & $\begin{array}{r}\text { DESIGN } \\
\text { BUILDER }\end{array}$ & 460-ONNCCE \\
\hline ORIGINAL & 4.1 & 6.8 & 4.1 & 2.168 & 1.932 & 0.461 & 0.518 \\
\hline ANÁLISIS & 0.0 & 6.8 & 8.2 & 2.168 & 1.932 & 0.461 & 0.518 \\
\hline
\end{tabular}

Tabla 2. Comparativa de valores "R" del ISOBLOCK. Fuente: Elaboración del autor.

Donde:

$\mathrm{Rt}=\mathrm{rsi}+\mathrm{R} 1+\mathrm{R} 2+\mathrm{R} 3+\mathrm{rse}=1 / \mathrm{hi}+\mathrm{L} 1 / \lambda 2+\mathrm{L} 2 / \lambda 2+\mathrm{L} 3 / \lambda 3+1 / \mathrm{he}$ $\mathrm{m} 2 \mathrm{~K} / \mathrm{W}$

En base a ese mecanismo, el resultado del cálculo de factor $\mathrm{R}$ es de $1.932 \mathrm{M} 2 \mathrm{~K} / \mathrm{W}$. La diferencia se debe a que no se consideran las juntas constructivas del material. Como se puede ver en la Tabla 2, el valor de "R" en el Isoblock es igual, independientemente de la ubicación del aislamiento.

Para la simulación térmica con Design Builder, se decidió alimentar el software para el cálculo de acuerdo a los datos de la conductividad térmica, densidad y calor específico que los proveedores del material facilitaron.

Para el módulo de block de concreto convencional, el factor $\mathrm{R}$ del material que se contempló fue de $1.11 \mathrm{~m}^{2} \mathrm{~K} /$ $W$, que es el que establece los ejemplos de la NMX-C-460ONNCCE-2009.

\section{RESULTADOS}

En la temporada de invierno, los resultados del estudio de campo arrojan que la temperatura del aire interior del módulo de Isoblock se encuentra un $62.02 \%$ dentro de la zona de confort, en tanto que el del módulo de block de concreto convencional sólo el $24.89 \%$. La temperatura del aire exterior se encuentra $31.86 \%$ del tiempo dentro de la zona de confort, lo que indica mejores condiciones que el módulo de block (ver Figura 7).

En la temporada de verano, los resultados del estudio de campo revelan que la temperatura del aire interior del módulo de Isoblock nunca se encuentra dentro de la zona de confort, en tanto que el del módulo de Block de concreto convencional lo hace el $17.92 \%$. La temperatura del aire exterior se encuentra $40.33 \%$ del tiempo dentro de la zona de confort, lo que indica mejores condiciones que el módulo de block e Isoblock en condiciones normales (ver Figura 8).

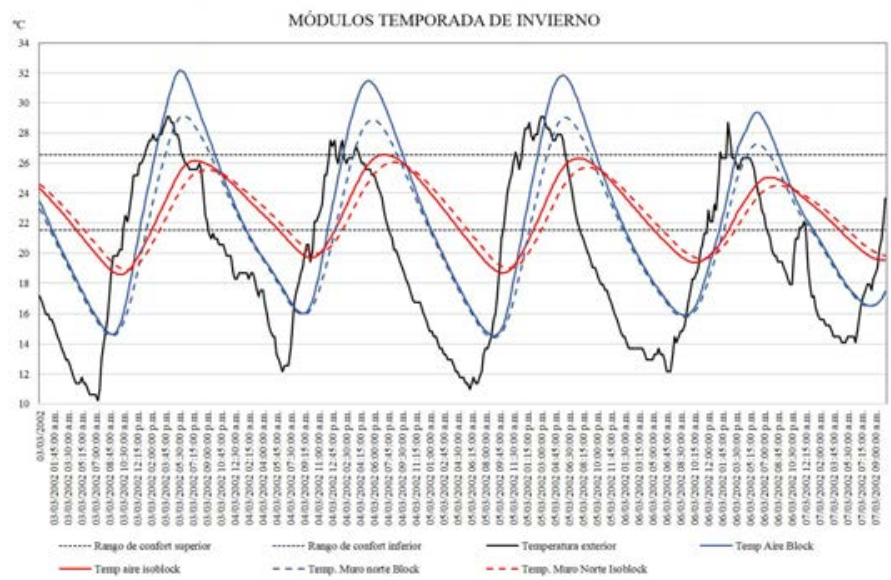

Figura 7. Resultados módulos de campo, temporada de invierno. Fuente: Elaboración del autor.

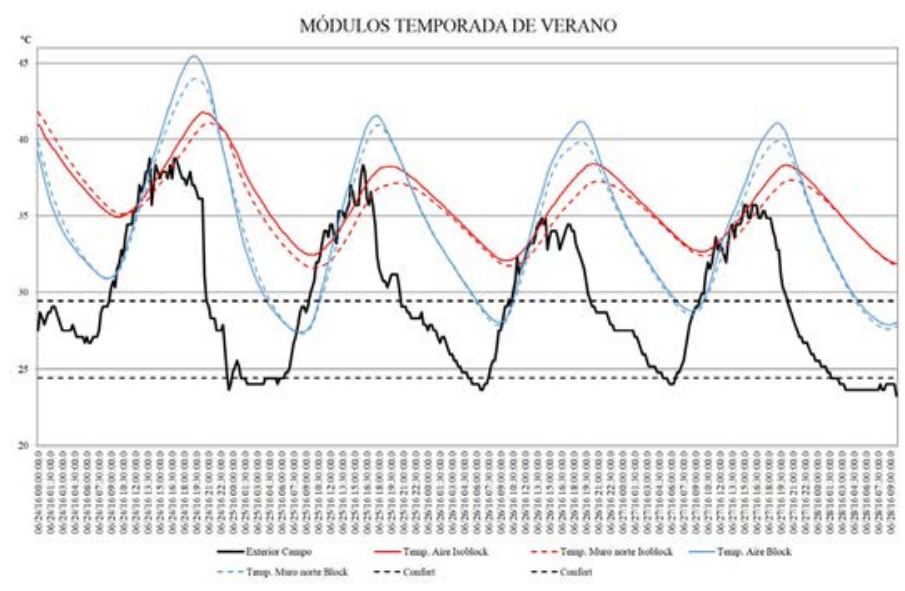

Figura 8. Resultados módulos de campo, temporada de verano. Fuente: Elaboración del autor. 


\begin{tabular}{|c|c|c|c|c|c|}
\hline & Exterior aire & Isoblock aire & Block aire & Block cable & Isoblock cable \\
\hline Máxima & 38.77 & 41.80 & 45.47 & 43.98 & 41.80 \\
\hline Promedio & 29.47 & 35.94 & 34.53 & 34.18 & 35.42 \\
\hline Mínima & 23.24 & 31.84 & 27.43 & 27.31 & 31.59 \\
\hline Oscilación & 15.53 & 9.95 & 18.04 & 16.67 & 10.21 \\
\hline
\end{tabular}

Tabla 3. Temperaturas del trabajo de campo. Temporada de verano. Fuente: Elaboración del autor.

La oscilación de temperatura del módulo de Isoblock es 44.84\% menor en comparación con la oscilación del módulo de block. Incluso, la temperatura del cable en el muro norte del Isoblock presenta un 38.75\% menos que el del block (ver Tabla 3), lo que demuestra la importancia de la resistencia térmica del material. No obstante, la diferencia de la oscilación térmica del aire indica que el efecto de la masa térmica mejora la eficiencia del muro.

Al obtener los resultados del comportamiento térmico del Isoblock en ambas temporadas y comprobar, en el periodo de verano, que hay un efecto de la masa térmica a considerar, se procedió a hacer simulaciones térmicas con la finalidad de evaluar en otras circunstancias los módulos. Para ello, lo primero que se hizo fue comparar las temperaturas exteriores registradas en campo con las que el software de Design Builder establece.

En ambas temporadas se puede observar un comportamiento similar en las gráficas. En la temporada de invierno, se aprecia que las temperaturas del Design Builder son menores en $7^{\circ} \mathrm{C}$ en promedio, durante todo el periodo de registro. En tanto, en la temporada de verano las diferencias son de $0.5^{\circ} \mathrm{C}$ en promedio (ver Figuras 9 y 10).

Para corroborar si lo evidenciado en la gráfica es factible de utilizar en las simulaciones, se procedió a aplicar una correlación de Pearson con los datos obtenidos registrados en el exterior y Design Builder en ambas temporadas de estudio.

En invierno, la correlación indica una $\mathrm{R}^{2}$ de 0.8163 , que significa una correlación de $R=0.903$, lo cual se considera como una correlación positiva muy alta (ver Figura 11).

En verano, la correlación señala una $\mathrm{R}^{2}$ de 0.81202 , que representa una correlación de $\mathrm{R}=0.901$, lo cual se considera, nuevamente, como una correlación positiva muy alta (ver Figura 12).

Los resultados de la simulación térmica con el software Design Builder en el periodo invernal indican que la temperatura del aire de los módulos está por debajo de la zona de confort. Y aunque en el estudio de campo los resultados del Isoblock sí se encontraban en la zona de confort, es necesario recordar que la diferencia de temperatura de los datos medidos con los del software tiene una diferencia de hasta $8^{\circ} \mathrm{C}$ menos, en el software (ver Figura 13).

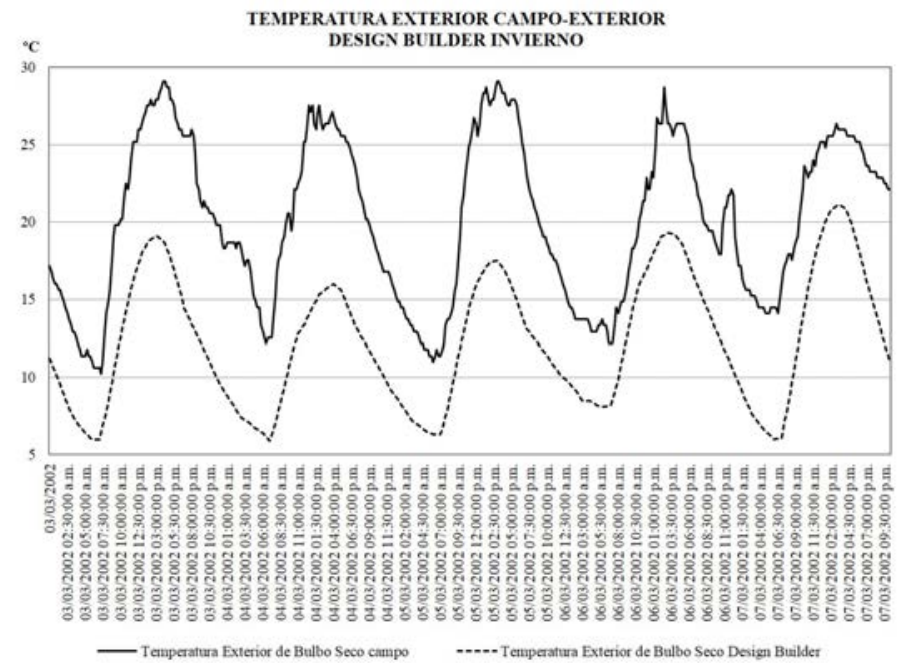

Figura 9. Comparación de temperaturas de invierno del exterior en campo y exterior Design Builder.

Fuente: Elaboración del autor.

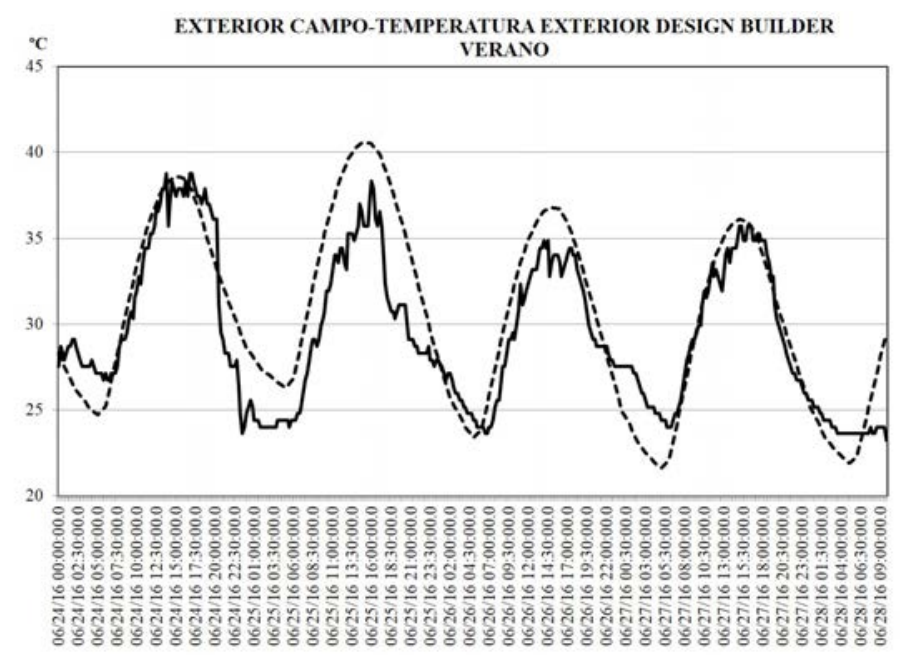

Figura 10. Comparación de temperaturas exterior de verano en campo y exterior Design Builder.

Fuente: Elaboración del autor. 


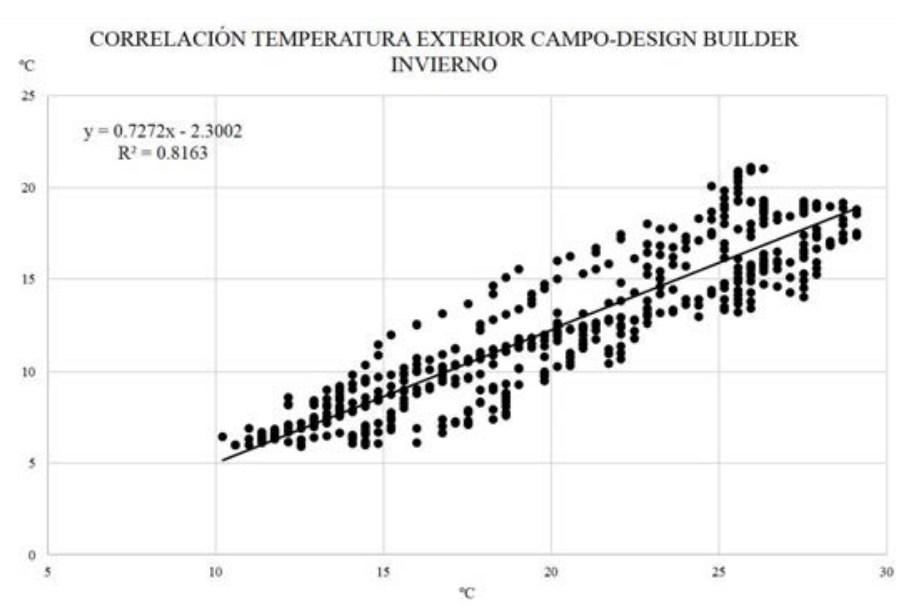

Figura 11. Correlación de Pearson de la temporada de invierno. Fuente: Elaboración del autor.

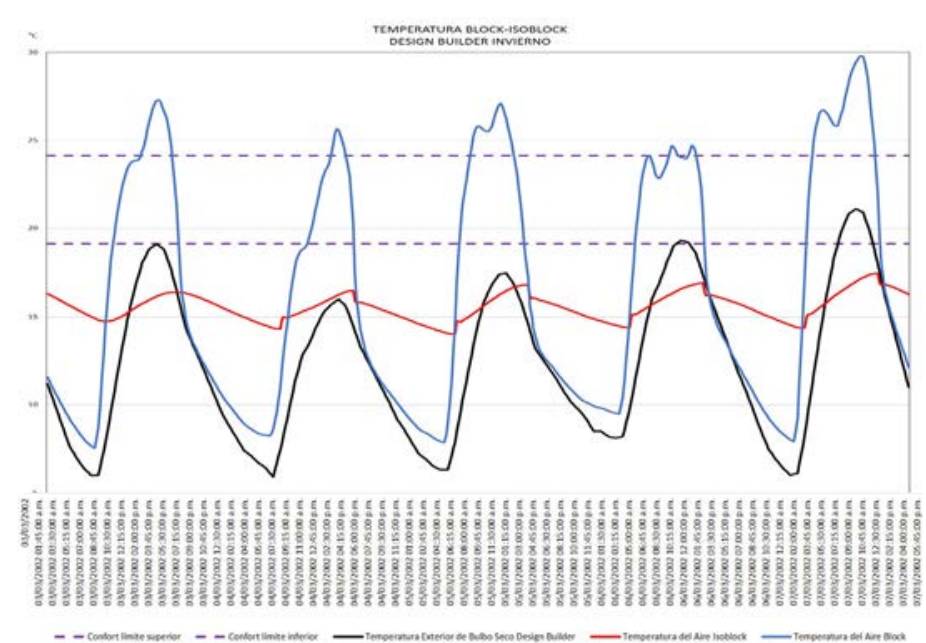

Figura 13. Resultados de la simulación térmica, temporada de invierno. Fuente: Elaboración del autor.

En el periodo estival la simulación revela que la temperatura del aire del módulo de lsoblock siempre se encuentra por encima de la zona de confort, al igual que en lo medido en campo. A diferencia de lo que ocurre en el módulo de block convencional, donde solo el $13.24 \%$ se encuentra en zona de confort. De la misma manera que en el estudio de campo, lo importante a destacar es la diferencia de la oscilación térmica del Isoblock y el block convencional, pues mientras que en el primero es de $1.56^{\circ} \mathrm{C}$, en el segundo es de $8.80^{\circ} \mathrm{C}$, lo cual es similar a lo observado en campo (ver Figura 14).

\section{EFECTO DE LA MASA TÉRMICA}

Al cambiar las proporciones de los concretos, como se mencionó en la Tabla 2, se observa claramente el efecto que tiene la inercia térmica en el comportamiento de la temperatura

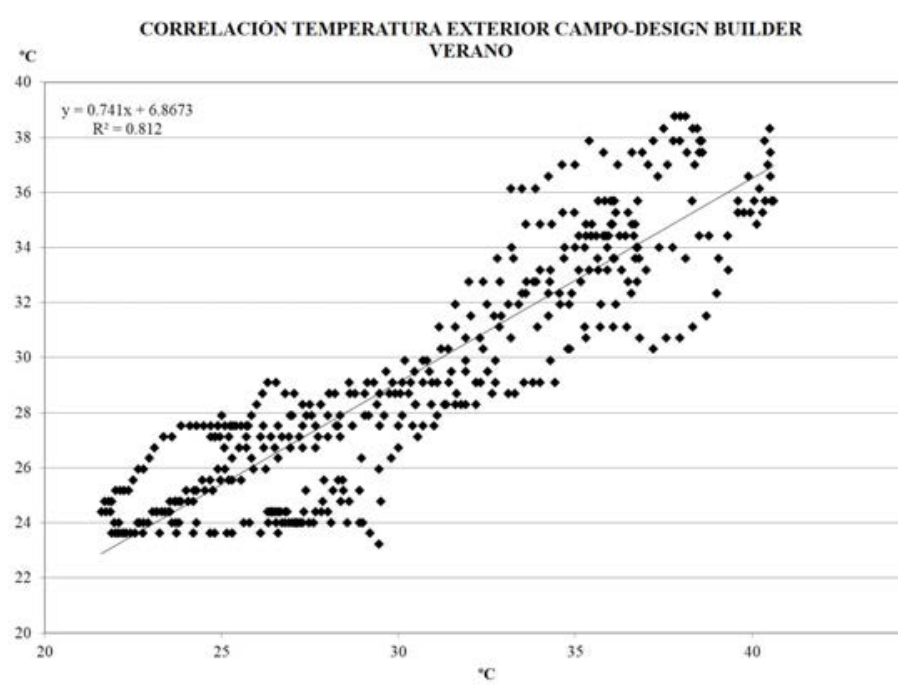

Figura 12. Correlación de Pearson de la temporada de verano. Fuente: Elaboración del autor.

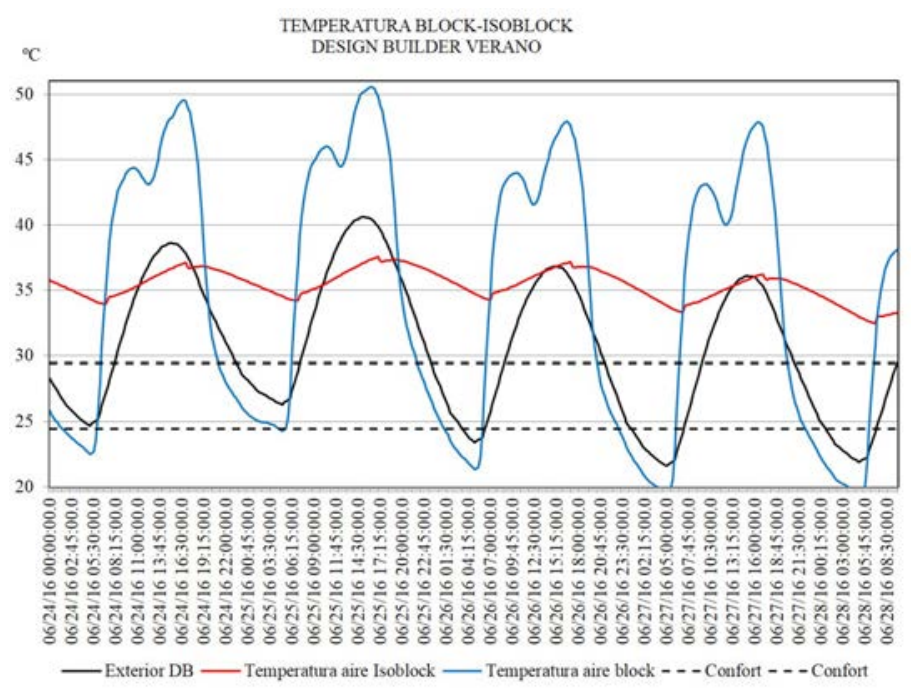

Figura 14. Resultados de la simulación térmica, temporada de verano. Fuente: Elaboración del autor.

del aire, en el módulo de Isoblock en invierno (ver Figura 15). Esta variación no considera ningún tipo de ganancia de calor extra.

De esta forma, se logra disminuir $0.28^{\circ} \mathrm{C}$ la temperatura máxima, mientras que la promedio aumenta $0.05^{\circ} \mathrm{C}$ y la mínima aumenta, también, $0.23^{\circ} \mathrm{C}$ (ver Figura 16). En concreto, la oscilación térmica del módulo disminuye de $3.45^{\circ} \mathrm{C}$ a $2.94^{\circ} \mathrm{C}$, es decir, $0.51{ }^{\circ} \mathrm{C}$, lo que significa un $14.78 \%$ de mejora (ver Tabla 4).

En verano es necesario ventilar para evitar el sobrecalentamiento que propician los materiales con un factor $\mathrm{R}$ alto y masa térmica, pues -como se observa en la Figura 14- si el espacio no está ventilado, la temperatura interior permanece por encima de la zona de confort. 


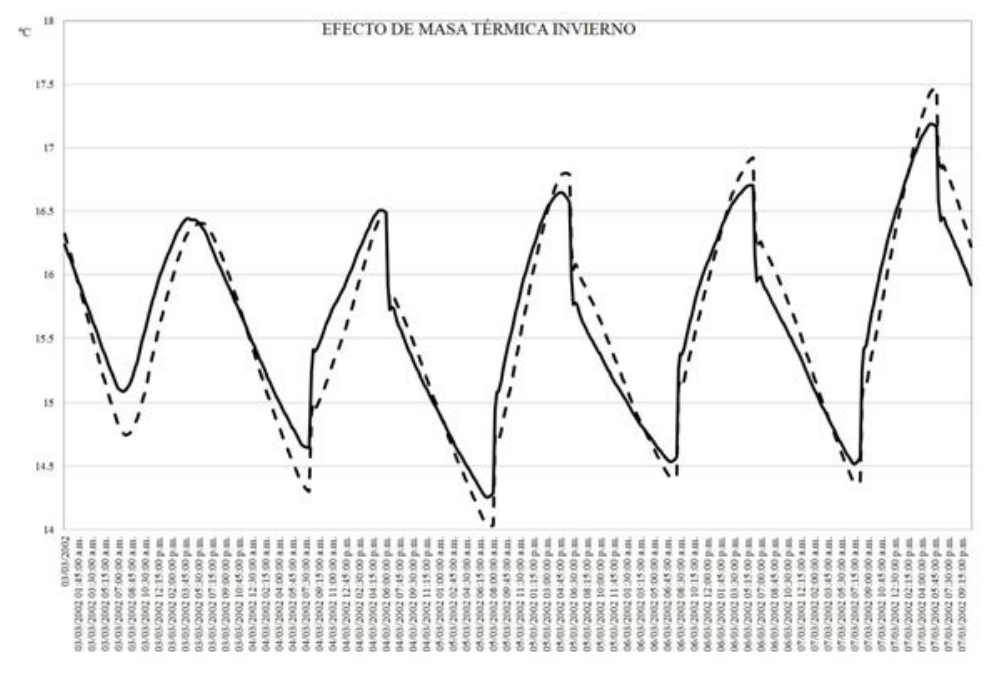

Figura 15. Efecto inercia térmica. Fuente: Elaboración del autor.

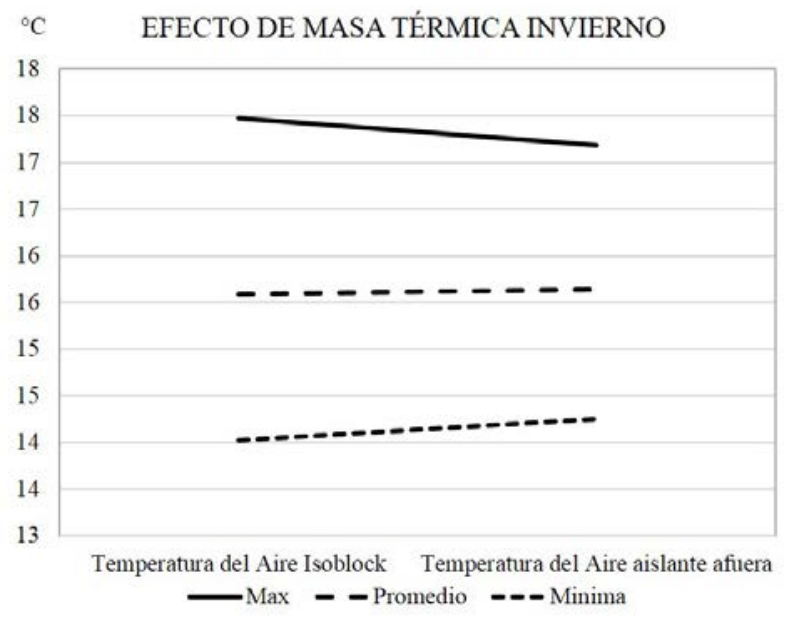

Figura 16. Efecto de la inercia térmica. Fuente. Elaboración del autor.

\begin{tabular}{|c|c|c|c|}
\hline & $\begin{array}{c}\text { Temperatura } \\
\text { Exterior }\end{array}$ & $\begin{array}{c}\text { Temperatura } \\
\text { del aire } \\
\text { Isoblock }\end{array}$ & $\begin{array}{l}\text { Temperatura } \\
\text { del aire } \\
\text { aislante afuera }\end{array}$ \\
\hline Max & 21.10 & 17.47 & 17.19 \\
\hline Promedio & 12.31 & 15.59 & 15.64 \\
\hline Mínima & 5.90 & 14.03 & 14.25 \\
\hline Oscilación & 15.20 & 3.45 & 2.94 \\
\hline
\end{tabular}

Tabla 4. Temperaturas del aire por efecto de la inercia térmica del ISOBLOCK en invierno. Fuente: Elaboración del autor.

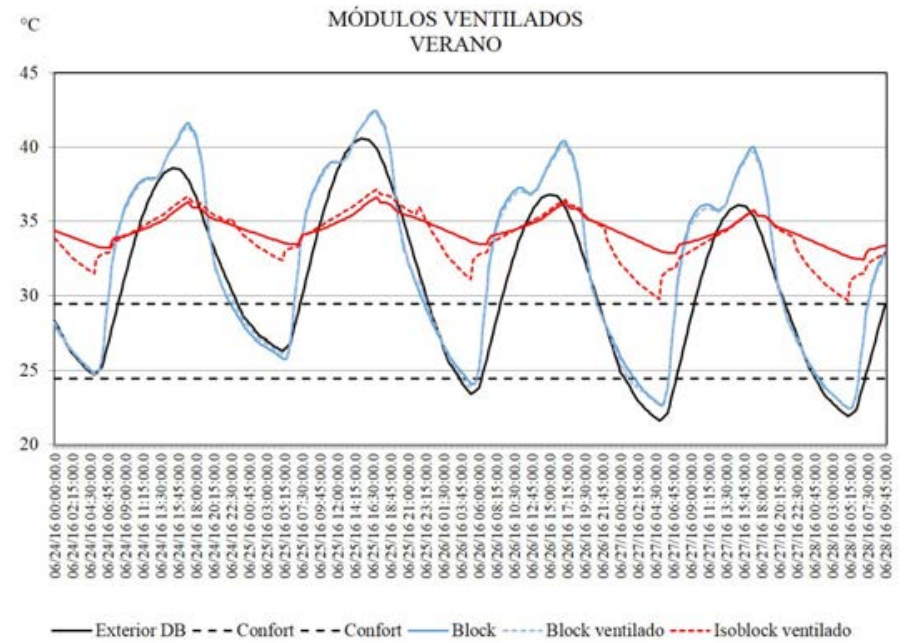

Figura 17. Efecto de la ventilación nocturna y masa térmica en verano. Fuente: Elaboración del autor.

La ventilación en este clima debe ser nocturna (enfriamiento convectivo nocturno) para aprovechar que la temperatura del aire baja acuciosamente durante la noche con respecto al día. Al utilizar esta técnica, es posible advertir que al ir pasando los días la temperatura del aire interior va disminuyendo paulatinamente, aunque sin alcanzar, en este caso, la zona de confort (ver Figura 17).

Se puede observar, asimismo, que la temperatura mínima del módulo ventilado de Isoblock disminuye en tanto que la máxima aumenta, lo que trae consigo la ampliación de la oscilación térmica diaria. El módulo de block ventilado no tiene variación en las temperaturas con respecto al que está sin ventilar, lo que confirma el aporte que tiene la masa térmica del concreto, en este caso aislado. 


\begin{tabular}{|c|c|c|c|c|c|}
\hline & Exterior & Block & $\begin{array}{c}\text { Block } \\
\text { ventilado }\end{array}$ & Isoblock & $\begin{array}{c}\text { Isoblock } \\
\text { ventilado }\end{array}$ \\
\hline Máxima & 40.60 & 42.45 & 42.31 & 36.64 & 37.15 \\
\hline Promedio & 30.50 & 32.15 & 32.06 & 34.43 & 33.89 \\
\hline Mínima & 21.60 & 22.44 & 22.40 & 32.45 & 29.65 \\
\hline Oscilación & 19.00 & 20.01 & 19.90 & 4.19 & 7.51 \\
\hline
\end{tabular}

Tabla 5. Temperaturas del aire por efecto de la inercia térmica del ISOBLOCK en verano. Fuente: Elaboración del autor.

\section{CONCLUSIONES}

Es necesario que la normativa mexicana haga una revaloración de la importancia de considerar la masa térmica de los materiales de la envolvente, pues si bien la resistencia térmica reduce la ganancia y/o pérdida de calor, esto no es suficiente para amortiguar las diferencias de temperatura del día y la noche en el clima cálido seco como el de Ciudad Juárez.

Se ha comprobado aquí que el Isoblock tiene un mejor desempeño térmico en invierno en comparación al aire exterior y el block de concreto, producto de su valor de resistencia térmica y del efecto producido por la masa térmica del material.

La temperatura del aire interior del módulo de block de concreto convencional se encuentra el $21.41 \%$ del tiempo, en promedio, dentro de la zona de confort. Mientras que la del módulo de Isoblock lo hace el 31.01\% del tiempo, en promedio. Ello evidencia que el Isoblock es $44.79 \%$ más eficiente que el block en verano, y $59.87 \%$, en invierno. En suma, es un $52.33 \%$ más en eficiente.

El uso de materiales con inercia térmica en invierno permite "almacenar calor" durante el día para que sea útil durante la noche, producto de su calor especifico volumétrico. Además, amplía el tiempo de retraso y el amortiguamiento del material, lo que reduce la pérdida de calor y dilata el tiempo en el que esto sucede.

En la temporada de verano, las cualidades térmicas de la inercia térmica pueden producir sobrecalentamiento al interior. Sin embargo, la aplicación de enfriamiento convectivo nocturno permite eliminar el calor acumulado durante el día en los materiales, pues si se ventilan los módulos, la eficiencia se incrementa hasta en un $80.4 \%$, lo que aumenta hasta el $71.21 \%$ la eficiencia con respecto al módulo con block convencional. Esto demuestra de manera contundente el efecto de la masa térmica en verano, siempre y cuando se ventile durante la noche.

Utilizar de manera adecuada los materiales de construcción de acuerdo a cada una de sus propiedades térmicas permite que las viviendas se acerquen a niveles de confort en cada una de las zonas climáticas. Ello reduce el uso de sistemas de climatización convencional con el consecuente ahorro energético y de recursos naturales.

Se puede evaluar otros espesores de masa térmica, incluso variando las dimensiones de los materiales estudiados, con miras a caracterizar, por medio de simulación las proporciones más adecuadas para cada una de las zonas térmicas del país.

\section{AGRADECIMIENTOS}

Se agradece al grupo Cementos de Chihuahua, que financió el proyecto de investigación, por la confianza de compartir los resultados.

\section{REFERENCIAS BIBLIOGRÁFICAS}

ANSI/ASHRAE Standard 55-2010. Thermal Environmental Conditions for Human Occupancy. American Society of Heating Refrigeration and Air-conditioning Engineers, 2010, pp. 11-12.

COMISIÓN NACIONAL DE VIVIENDA (CONAVI). Soluciones verdes para el sector vivienda. México: CONAVI, 2010.

COMISIÓN NACIONAL DEL AGUA (CONAGUA). Servicio Meteorológico Nacional, Normales Climatológicas por Estación [en línea]. [Consultado 25 junio 2016]. Disponible en: www.smn. cna.gob.mx

FUNDACIÓN IDEA (para la implementación, diseño, evaluación y análisis de política públicas A.C.). Estrategia Nacional para la Vivienda Sustentable. México: Fundación IDEA A.C., 2013.

GRUPO CEMENTOS DE CHIHUAHUA. Isoblock GCC, block térmico de concreto [en línea], 2016. [Consultado 20 agosto 2017]. Disponible en: http://www.gcc.com/wp-content/uploads/2016/08/ ISOBLOCK-Folleto-0817_digital-final.pdf

NMX-C-460-ONNCCE-2009. Industria de la construcciónaislamiento térmico-valor " $\mathrm{R}$ " para las envolventes de vivienda por zona térmica para la República Mexicana-especificaciones y verificación. Secretaría de Economía. 30 julio 2009. México D.F.

SEMARNAT. Secretaría de Medio Ambiente y Recursos Naturales. Gobierno de México. Blog [en línea]. Actualizada: 10 diciembre 2016. [Consultado 11 diciembre 2017]. Disponible en: https:// www.gob.mx/semarnat/articulos/protocolo-de-kioto-sobre-cambioclimatico?idiom $=\mathrm{es}$

VALLEJO, Víctor. Las diversas certificaciones aplicables a los edificios sustentables en México. Multidisciplinar [en línea], 2014, n 18, pp. 29-58. [Consultado 25 agosto 2017]. Disponible en:http://www.Acatlan.unam.mx/file_download/2081/ multi-2014-05-02.pdf 